\title{
Transepithelial Photorefractive Keratectomy with Crosslinking for Keratoconus
}

\author{
Achyut N. Mukherjee*, Vasilis Selimis and Ioannis Aslanides
}

Emmetropia Mediterranean Eye Clinic, Elefteria Square 44, Heraklion, Crete 71201, Greece

\begin{abstract}
Purpose: To analyse visual, refractive and topographic outcomes of combining transepithelial photorefractive keratectomy (tPRK) with simultaneous corneal crosslinking for the visual rehabilitation of contact lens intolerant keratoconus patients.

Methods: Patients with topographically significant keratoconus, limited corrected vision and intolerant of contact lenses were prospectively recruited, subject to ethical approval and consent. All patients underwent single step aspheric tPRK and sequential crosslinking. Preoperative vision, refraction, corneal topography and wavefront were assessed, with postoperative assessment at $1,3,6$, and 12 months.

Results: 22 eyes of 14 patients were included in the pilot study. Mean age was 32 years (SD 6.8, range 24 to 43 ). Mean preoperative unaided vision was 1.39 LogMAR (SD 0.5) best corrected 0.31 LogMAR (SD 0.2). Mean preoperative spherical equivalent was -2.74 Diopters (D) (SD 4.1 range -12.25 to +7.75 ), and mean cylinder $-2.9 \mathrm{D}$ (SD 1.2, range 0 to -5.5). Mean central corneal thickness was 461um (SD 29, range 411 to 516). Vision improved postoperatively; unaided 0.32 LogMAR (SD 0.4), best corrected 0.11 (SD 0.13) $(\mathrm{P}=<0.005)$. Mean postoperative cylinder was $-1.4 \mathrm{D}$ (SD1.2), significantly reduced $(\mathrm{p}<0.005)$. Maximum keratometry $(\mathrm{Kmax})$ was stable throughout postoperative follow up. $(\mathrm{p}<0.05)$.

Conclusions: Non topographic transepithelial PRK with simultaneous crosslinking improves vision, and may offer an alternative to keratoplasty in contact lens intolerant keratoconus. Further comparative studies to topographic PRK techniques are indicated.
\end{abstract}

Keywords: Keratoconus, photorefractive keratectomy, collagen crosslinking therapy (CXL), tPRK.

\section{INTRODUCTION}

Keratoconus is the most common keratectasia, where thinning and irregularity of the cornea result in visual disability, with often profound impact on young patients. Current mainstream therapy is the use of rigid contact lenses, with keratoplasty reserved for advanced cases or contact lens intolerance. Emerging therapies include intrastromal ring segments and photorefractive keratectomy (PRK) in addition to collagen crosslinking therapy (CXL), which aims to stabilize rather than improve visual function [1]. Current regimes for PRK utilize topographically guided profiles, intended to regularise the corneal surface and improve vision [2-9].

The aim of the current study was to evaluate a novel transepithelial PRK protocol where, in contradiction to previous studies, topographic treatment planning and profiles were not used. This was combined with simultaneous CXL. This treatment was utilized for contact lens intolerant patients with visually disabling keratoconus, for whom further conventional therapy would involve keratoplasty.

*Address correspondence to this author at the Emmetropia Mediterranean Eye Clinic, Elefteria Square 44, Heraklion, Crete 71201, Greece; Tel: 0030 2810 226198; Fax. 00302810343436 ;

E-mail: hatch.mukherjee@gmail.com

\section{PATIENTS \& METHODS}

This was a prospective uncontrolled interventional pilot study, consisting of 22 eyes of 14 consecutive patients with keratoconus prospectively recruited at a private eye clinic in Crete, Greece, who underwent combined simultaneous transepithelial PRK and CXL.

Inclusion criteria were a minimum age of 21 years, clinical evidence of keratoconus, as well as topographic changes consistent with moderate keratoconus severity (Amsler-Krumeich stage I-II). All patients had visually significant disease, with significant disability not adequately corrected with spectacles and best spectacle corrected vision worse than 0.8 decimal Snellen fraction (0.1 LogMAR equivalent). All cases were either intolerant of rigid contact lenses, or unable to wear contact lenses due to social or occupational reasons. Patients were included irrespective of whether the keratoconus was stable or progressive. Cases were excluded in the presence of any significant corneal scarring or hydrops, co-existing ocular pathology or previous surgery. Patients were prospectively recruited subject to informed consent. Ethical approval for the study was sought and obtained from the institutional research ethics committee.

Preoperative assessment included uncorrected and spectacle corrected acuity, cycloplegic and non cycloplegic autorefraction and subjective refraction, and slit lamp 
examination including dilated fundoscopy and intraocular pressure. Corneal topography with Orbscan IIz (Bausch \& Lomb, USA), Keratron Scout corneal wavefront analysis (Optikon S.p.A. Italy), Hartmann Schack Aberrometery (ORK-Wavefront-Analyzer, Schwind eye-tech-solutions $\mathrm{GmbH}$, Germany), and ultrasound pachymetry (CorneoGage plus, Sonogage, USA) were also assessed.

\section{SURGICAL PLANNING \& TECHNIQUE}

All treatments were carried out using the Schwind AMARIS 750 excimer laser platform (Schwind eye-techsolutions $\mathrm{GmbH}$, Kleinostheim, Germany), a 193nm, $0.54 \mathrm{~mm}$ super Gaussian profile flying spot laser with 6 axis eye tracking, and thermal optimisation. In contrast to previous studies, we utilized a transepithelial single step PRK (All Surface Laser Ablation or ASLA) with a population based custom epithelial profile and reverse application of the refractive ablation first followed by the epithelial component, in an uninterrupted delivery [10]. This was followed by immediate CXL. Unlike some previous studies, Mitomycin C was not used [2,3]. All laser profiles were based on spherocylindrical parameters only, and were not topography or wavefront guided as in previous studies [2-5]. Laser surgical parameters were determined according to the following algorithm. The optical zone was reduced to minimise ablation depth depending on the planned correction, typically $5.5 \mathrm{~mm}$. The subjective refractive cylinder was corrected up to $100 \%$, but generally a lower proportion, up to a maximal ablation depth over the ectatic cornea of 50um. This was correlated to the topographic cylinder. In cases where the topographic and subjective cylinder did not correlate, a lower proportion of cylinder was corrected. If the cylindrical correction did not require the maximal permitted 50um ablation, then the remaining available ablation depth was used to correct as much spherical error as possible.

Prior to ablation a Merocell sponge (Medtronic Inc., USA) dipped in balanced salt solution (BSS) was applied on the corneal surface to avoid uneven wetting and hence ablation [10]. Following single step excimer laser ablation, the cornea was cooled with $20 \mathrm{ml}$ chilled BSS and ultrasonic pachymetry of the residual thickness was performed. Riboflavin drops were applied, using hypo-osmolar drops in cases with <350um residual pachymetry for 5 minutes. Immediate corneal crosslinking was then carried out using a UV-X Crosslinker (Peshke Meditrade $\mathrm{GmBH}$ ) with a radiation of $3 \mathrm{~mW} / \mathrm{cm}^{2}$ for 30 minutes. 1 drop of topical Ketorolac $0.5 \%$ and 1 drop of Ofloxacin $0.3 \%$ were instilled and a bandage contact lens inserted. Postoperative drops were Ofloxacin $0.3 \%$ q.i.d. until contact lens removal, Dexamethasone $0.1 \%$ drops q.i.d., and artificial tear drops q.i.d.

\section{Postoperative Assessment}

Patients underwent routine postoperative assessment at day $1,3,7$ and month 1, 3, 6 and 12. At the postoperative study visits after 3 months, vision, subjective refraction, slitlamp biomicroscopy with grading of haze and corneal topography were performed.

\section{Analysis}

Data were analysed using Microsoft Excel (Microsoft Corporation, Redmond, WA, USA). Continuous variables were compared using unpaired t-tests, with temporal changes using paired t-tests. Categorical data were compared using Fishers exact test.

\section{RESULTS}

22 eyes of 14 consecutive patients were included, subject to meeting the inclusion criteria and informed consent. There were 10 male and 4 female patients. Mean age was 32 years (SD 6.8, range 24 to 43 ).

\section{Treatment Parameters}

Due to the variable proportion of total refractive error treated as noted above, the relative proportion corrected was assessed for each case. The mean proportion of preoperative astigmatic error targeted in the ablation profile planning was $77.1 \%$ (SD25.7). The mean spherical correction was $58.7 \%$ (SD 51.1) of the full refractive spherical error. Preoperative ultrasound central corneal thickness was 461 um (SD 27, range 411 to 516). There were no adverse events.

\section{Visual Acuity}

Mean preoperative BCVA was 0.31 LogMAR (SD 0.2, range 0.1 to 0.7 ). Mean BCVA at 6 months was 0.23 (SD 0.22 range 0.05 to 0.7 ) and at 12 months follow up was 0.11 LogMAR (SD 0.13, range -0.08 to 0.5 ) ( $\mathrm{p}<0.0001$ compared to preoperative). Mean preoperative UCVA was 1.39 LogMAR (SD 0.5 , range 0.3 to 1.7 ) and postoperatively at 6 months was 0.36 (SD 0.46 range 0.05 to 1.7 ), at 12 months improving to $0.27 \operatorname{LogMAR}$ (SD 0.4, range 0 to 1.7), significantly better than baseline $(\mathrm{p}<0.0001)$. Functionally, at 12 months, $64 \%$ of eyes achieved 0.1 LogMAR (Snellen $6 / 8.5$ ) or better postoperatively vs $22 \%$ achieving the same level of acuity preoperatively $(\mathrm{p}=0.021)$, while $41 \%$ achieved 0.05 LogMAR (Snellen 6/6.7) compared to none preoperatively. The change of visual acuity distribution pre to post operatively is shown in Fig. (1). In terms of safety, no eye lost lines of best corrected acuity. $68 \%$ of eyes improved by 2 or more lines of corrected acuity postoperatively (Fig. 2).

\section{Refractive Outcome}

There was a statistically significant reduction in spherical equivalent refraction from mean preoperative $-2.74 \mathrm{D}(\mathrm{SD}$ 4.0, range -12.25 to 7.75$)$ to 12 months postoperative mean $0.3 \mathrm{D}$ (SD 3.2, range -5.0 to +9.0$)(\mathrm{P}=0.002)$. Mean absolute spherical equivalent was 3.88D (SD 2.98, range 0 to 12.25) preoperatively and postoperatively was $1.99 \mathrm{D}$ (SD 2.5, range 0 to +9.0$)(\mathrm{p}=0.019)$. Mean spherical equivalent prediction error (achieved - expected spherical equivalent) was $1.3 \mathrm{D}$ (SD 2.68, range -4.75 to +10 ). Mean preoperative refractive 


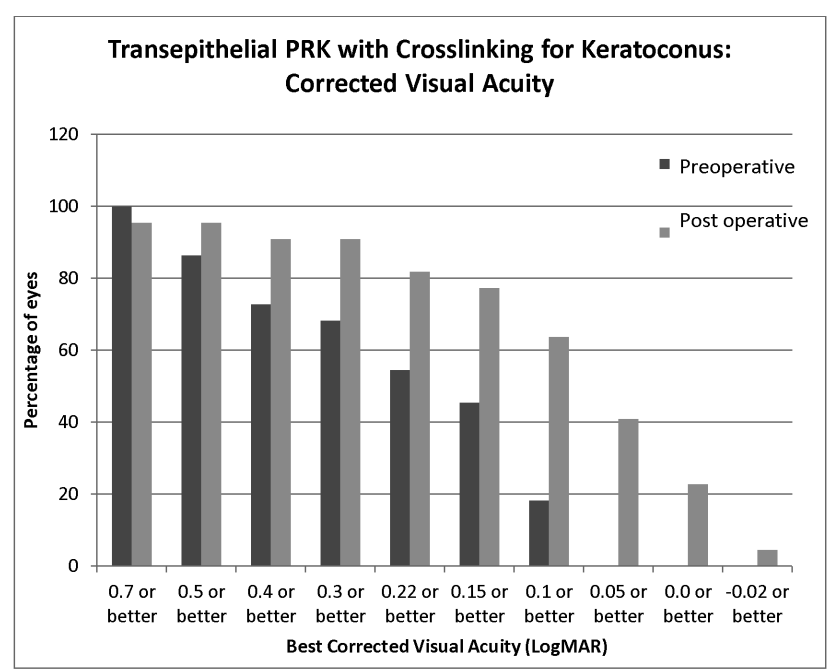

Fig. (1). Postoperative improvement in corrected visual acuity at 12 months following transepithelial non topographic photorefractive keratectomy in contact lens intolerant keratoconics.

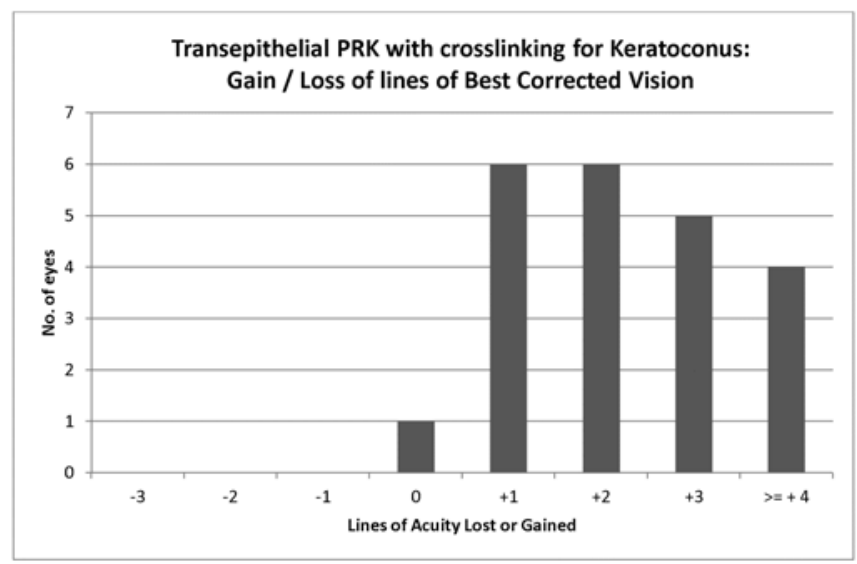

Fig. (2). Postoperative safety and improvement in lines of acuity at 12 months following transepithelial non topographic photorefractive keratectomy for keratoconus.

astigmatism was $-2.95 \mathrm{D}$ (SD 1.2, range -5.5 to 0 ), and postoperatively significantly decreased to $-1.45 \mathrm{D}$ (SD 1.2, range -3.5 to 0$)(p=0.0009)$ (Fig. 3).

\section{Topographic Parameters}

Keratometric values were significantly reduced at the 12 month visit, from mean keratometry (Kmean) 46.7D (SD 3.5, range 40.3 to 55.3) and mean steepest keratometry (Kmax) 48.6 (SD 3.7, range 41.5 to 57.9) preoperatively to Kmean 44.8D (SD 2.2, range 42.2 to 49.8) and Kmax 44.46D (SD 3.1, range 39.2 to 53.0) postoperatively, a statistically significant reduction $(\mathrm{P}=0.0004, \quad \mathrm{P}=0.001$ respectively). We further sought to assess the stability of the treatment over time. The variation in Kmax over time is shown in Fig. (4), with associated $\mathrm{P}$ values compared to preoperative baseline and 3 month postoperative visit. Keratometric values appeared stable postoperatively, with no evidence of progression in Kmax over the follow up period ( $p>0.05$ at all time points). Preoperative mean keratometric astigmatism was $3.85 \mathrm{D}$ (SD 1.93 , range 0.3 to 9.0 ), postoperatively at $3.48 \mathrm{D}(\mathrm{SD} 2.03$, range 0.3 to 7.4$)(\mathrm{P}=$ 0.33 ). Pre and postoperative topographic characteristics of a typical treatment are shown in Fig. (5).

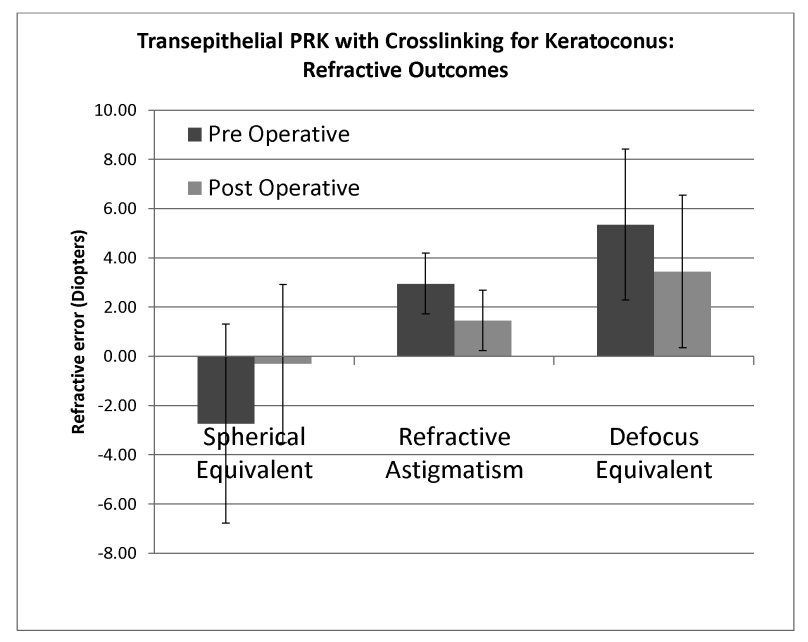

Fig. (3). Refractive changes at 12 months following transepithelial non topographic photorefractive keratectomy for keratoconus.

\section{Haze}

Haze was clinically graded according to the scheme of Fantes et al. [11]. 4 (18\%) of eyes developed some haze, with 1 eye grade 1,2 grade 2 and 1 grade 3 . Only the eye with grade 3 haze was felt to have any possible impact on vision, although this eye still had a vision of 0.1 LogMAR compared to 0.3 preoperatively, and was continuing to regress at last follow up.

\section{DISCUSSION}

Corneal crosslinking therapy has been a paradigm change in keratoconus management. It is not, however, primarily intended to correct existing visual deficit. The use of rigid contact lenses is for the most part an acceptable option. However for a significant number of patients these may not be tolerable in terms of comfort, limited wear time, occupational or social limitations [12]. Additionally, a proportion of patients do not tolerate rigid contact lens wear at all, necessitating keratoplasty, but this is limited by prolonged rehabilitation, risks, visual limitation, and recurrent need for rigid lenses to achieve functional vision [13]. Several newer therapies have been reported in combination with cross linking to address the need for visual rehabilitation in this group, including the use of intracorneal ring segments, and more recently excimer laser PRK surface ablation [3,5-7,14,15]. In this series we assessed a nontopographic protocol for the latter.

The most notable finding of our series was a significant improvement in vision, both corrected and unaided. The proportion of eyes achieving a functional threshold of 0.1 LogMAR (Snellen 6/8.5) increased from 22 to $64 \%$, a significant proportion who might otherwise have to consider keratoplasty. Notably, even in cases with residual refractive errors, these were easily correctable with glasses or soft contact lenses rather than rigid lenses, with benefits of wear 
Temporal Changes in Keratometry (Kmax) Following Transepithelial Photorefractive Keratectomy with Crosslinking for Keratoconus

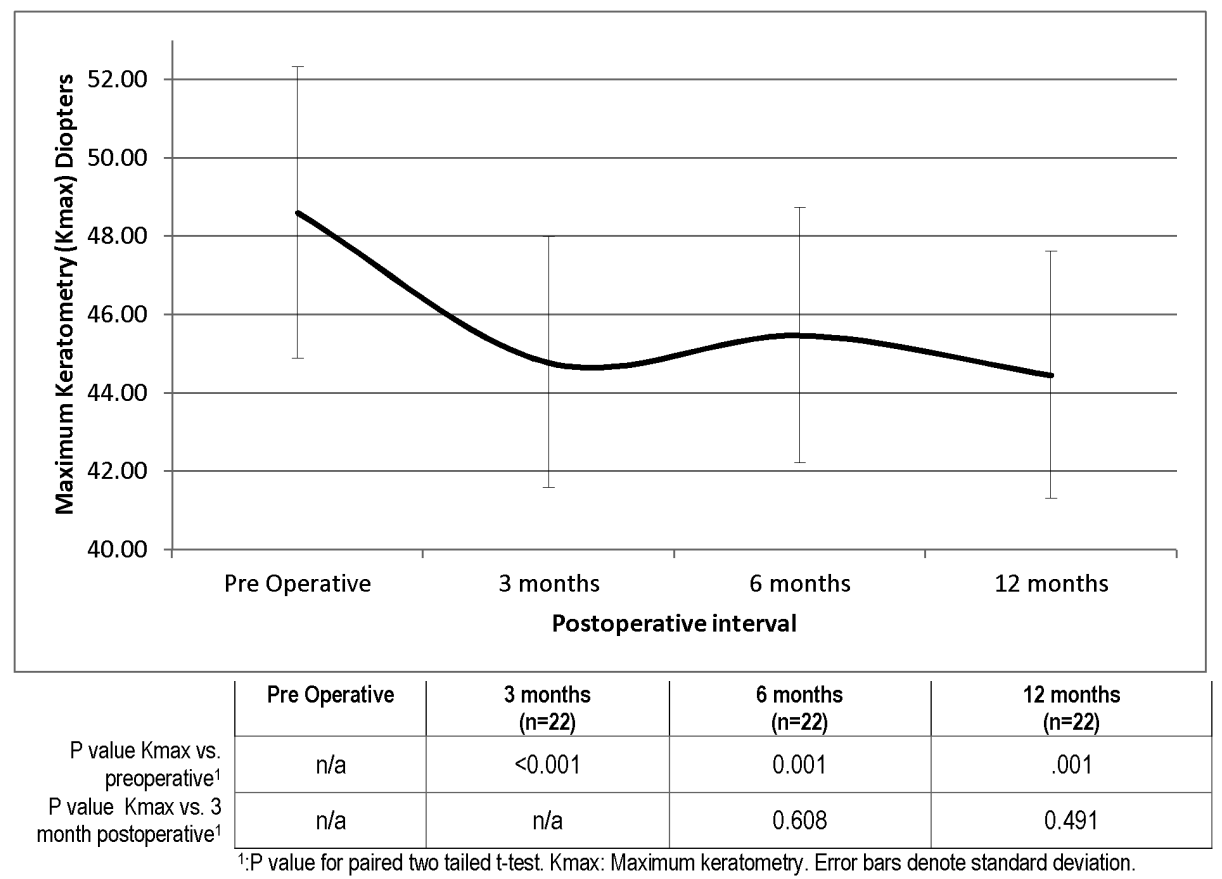

Fig. (4). Temporal Changes in Keratometry (Kmax) Following Non-topographic Transepithelial Photorefractive Keratectomy with Crosslinking for Keratoconus. The adjoined table provides the statistical assessment of changes compared to preoperative (first row) and over the post operative period (second row), showing a statistically significant reduction postoperatively, but stability over the postoperative follow up period.

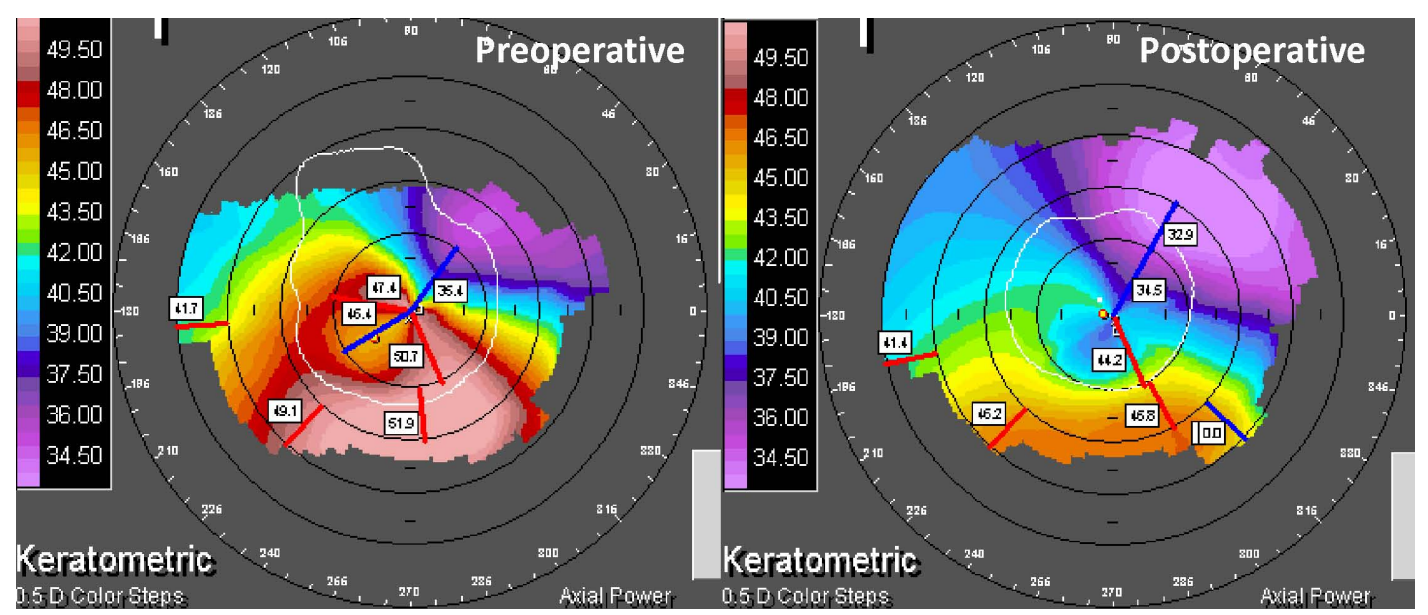

Fig. (5). Clinical example of non-topographic transepithelial photorefractive keratectomy with combined crosslinking for keratoconus. Preoperative and postoperative corneal topography at 24 months. Vision improved from LogMAR 1.0 unaided and 0.7 best corrected to 0.05 unaided, -0.08 best corrected.

time and tolerance. These visual acuity improvements compare favourably to those described in published series of topographically guided excimer treatments. By comparison current studies of CXL alone demonstrate a modest and unpredictable improvement in visual function in just over half of cases, with the remainder remaining stable, but a proportion also worsening in acuity terms [16, 17]. Correction of topographic parameters was not directly targeted by the technique; however there was significant improvement in topographic parameters. This change in parameters was unpredictable and greater than expected given the ablation profiles, highlighting the unpredictable nature of topographic response in ectasia. A concern related to the use of PRK in keratoconus is the possibility of accelerating or inducing disease progression. However even without CXL, Alpins et al. followed patients up to 10 years after PRK for keratoconus with no evidence of progression, while Cennamo et al. found no progression at 2 years $[7,9]$. 
Kannelopoulos et al. followed patients with combined CXL and PRK for a mean of 3 years, with no obvious increased progression, while in a smaller series Stjanovic et al. also found no progression at a year, as did Kymionis et al. $[3,5,15]$. Alessio et al., comparing combined PRK with CXL to CXL alone, found no evidence of increased progression at 2 years [18]. We found no clinical suggestion of progressive ectasia in any eye, despite not excluding progressive disease from the initial study cohort. Although limited by the follow up period, there was some evidence of keratometric stability in Kmax over this period.

In this study we utilized non-topographic aspheric profiles derived from refraction and topography, combined with simultaneous CXL. In contrast, current treatment strategies use topographically derived treatment profiles with the intention of correcting aberrations. The use of topographic profiles however, assumes that the shape change in the ectatic cornea predictably responds to ablation shape. However, there are several potential causes of unpredictable response to topographic planning. Firstly, the use of topographic profiles ignores the biomechanical effects of such ablations in compromised cornea [19]. Secondly, the actual tissue ablation rate may differ in keratoconic tissue, with unexpected topographic effects. Thirdly, the measured and ablated surfaces differ unless a transepithelial therapy is used, since the epithelial profile in keratoconus is irregular [5]. Finally and most significantly, crosslinking itself has progressive effects on keratoconic topography, which are not accounted for $[16,20]$.

Our rationale for non-topographic aspheric astigmatic profiles was minimise stromal ablation, while preserving beneficial effects of combining PRK and CXL. A transepithelial treatment was chosen to obtain a corrective effect based on the differential epithelial profile around the cone, which has been shown to have a 'doughnut' distribution, thus the masking effect of the epithelium would tend to regularise the cone [21]. Notably, combined excimer laser ablation and CXL may also have a beneficial effect on keratoconus independent of topographic or refractive correction. This has been demonstrated in a recent series by Kymionis et al., where PTK epithelial removal for CXL resulted in superior visual outcomes compared to mechanical removal [22]. Although MMC in keratoconic PRK has been used, we felt that CXL induced keratocyte depopulation offsets the need for chemotherapy, particularly as the transepithelial treatment causes less haze [10]. Although we had initial concerns regarding potential haze, in this and a previous transepithelial PRK study, haze was insignificant [5].

The major limitation of the study was the absence of a control group. We did not feel that a retrospective control group of CXL alone would be superior to comparison of existing large CXL series. A future study comparing topographic to non-topographic treatment is planned. Notwithstanding these issues, we feel that this technique shows promise as a preliminary intervention in contact lens intolerant keratoconics with moderate disease, particularly where keratoplasty or other surgical intervention is being considered, and is worthy of further evaluation.

\section{CONFLICT OF INTEREST}

The authors confirm that this article content has no conflict of interest.

\section{ACKNOWLEDGEMENTS}

Declared none.

\section{REFERENCES}

[1] Wollensak G, Spoerl E, Seiler T. Riboflavin/ultraviolet-a-induced collagen crosslinking for the treatment of keratoconus. Am J Ophthalmol 2003; 135: 620-7.

[2] Kanellopoulos AJ, Binder PS. Collagen cross-linking (CCL) with sequential topography-guided PRK: a temporizing alternative for keratoconus to penetrating keratoplasty. Cornea 2007; 26: 891-5.

[3] Kanellopoulos AJ. Comparison of sequential vs same-day simultaneous collagen cross-linking and topography-guided PRK for treatment of keratoconus. J Refract Surg 2009; 25: S812-8.

[4] Kymionis GD, Portaliou DM, Kounis G a, et al. Simultaneous topography-guided photorefractive keratectomy followed by corneal collagen cross-linking for keratoconus. Am J Ophthalmol 2011; 152: 748-55.

[5] Stojanovic A, Zhang J, Chen X, et al. Topography-guided transepithelial surface ablation followed by corneal collagen crosslinking performed in a single combined procedure for the treatment of keratoconus and pellucid marginal degeneration. J Refract Surg 2010; 26: 145-52.

[6] Mortensen J, Ohrström a. Excimer laser photorefractive keratectomy for treatment of keratoconus. J Refract Corneal Sur 1994; 10: 368-72.

[7] Alpins N, Stamatelatos G. Customized photoastigmatic refractive keratectomy using combined topographic and refractive data for myopia and astigmatism in eyes with forme fruste and mild keratoconus. J Cataract Refract Surg 2007; 33: 591-602.

[8] Tamayo GE, Serrano MG. Treatment of irregular astigmatism and keratoconus with the VISX C-CAP method. Int Ophthalmol Clin 2003; 43: 103-10.

[9] Cennamo G, Intravaja A, Boccuzzi D, et al. Treatment of keratoconus by topography-guided customized photorefractive keratectomy: two-year follow-up study. J Refract Surg 2008; 24: 145-9.

[10] Aslanides IM, Padroni S, Mosquera SA, et al. Comparison of single-step reverse transepithelial all-surface laser ablation (ASLA) to alcohol-assisted photorefractive keratectomy. Clin Ophthalmol 2012; 6: 973-80.

[11] Fantes FE, Hanna KD, Waring GO, et al. Wound healing after excimer laser keratomileusis (photorefractive keratectomy) in monkeys. Arch Ophthalmol 1990; 108: 665-75.

[12] Erdurmus M, Yildiz EH, Abdalla YF, et al. Contact lens related quality of life in patients with keratoconus. Eye Contact Lens 2009; 35: 123-7.

[13] Jones MNA, Armitage WJ, Ayliffe W, et al. Penetrating and deep anterior lamellar keratoplasty for keratoconus: a comparison of graft outcomes in the United kingdom. Invest Ophthalmol Vis Sci 2009; 50: 5625-9.

[14] Koller T, Iseli HP, Donitzky C, et al. Topography-guided surface ablation for forme fruste keratoconus. Ophthalmology 2006; 113 : 2198-202.

[15] Kymionis GD, Kontadakis GA, Kounis GA, et al. Simultaneous topography-guided PRK followed by corneal collagen cross-linking for keratoconus. J Refract Surg 2009; 25: S807-11.

[16] Raiskup-Wolf F, Hoyer A, Spoerl E, et al. Collagen crosslinking with riboflavin and ultraviolet-A light in keratoconus: long-term results. J Cataract Refract Surg 2008; 34: 796-801.

[17] Kolli S, Aslanides IM. Safety and efficacy of collagen crosslinking for the treatment of keratoconus. Expert Opin Drug Saf 2010; 9: 949-57.

[18] Alessio G, L'abbate M, Sborgia C, et al. Photorefractive keratectomy followed by cross-linking versus cross-linking alone for management of progressive keratoconus: two-year follow-up. Am J Ophthalmol 2013; 155: 54-65.e1. 
[19] Dupps WJ, Wilson SE. Biomechanics and wound healing in the cornea. Exp Eye Res 2006; 83: 709-20.

[20] $\mathrm{Tu} \mathrm{KL}$, Aslanides IM. Orbscan II anterior elevation changes following corneal collagen cross-linking treatment for keratoconus. J Refract Surg 2009; 25: 715-22.

[21] Reinstein DZ, Gobbe M, Archer TJ, et al. Epithelial, stromal, and total corneal thickness in keratoconus: three-dimensional display with artemis very-high frequency digital ultrasound. J Refract Surg 2010; 26: 259-71.

[22] Kymionis GD, Grentzelos M a, Kounis G a, et al. Combined transepithelial phototherapeutic keratectomy and corneal collagen cross-linking for progressive keratoconus. Ophthalmology 2012; 1 8.

Received: August 7, 2013

Revised: September 28, 2013

Accepted: October 2, 2013

(C) Mukherjee et al.; Licensee Bentham Open.

This is an open access article licensed under the terms of the Creative Commons Attribution Non-Commercial License (http://creativecommons.org/licenses/by$\mathrm{nc} / 3.0 /$ /) which permits unrestricted, non-commercial use, distribution and reproduction in any medium, provided the work is properly cited. 